\title{
Exploring the Innovation, Integration and Lead of the Cultural and Sentimental Program: A Case Study of The Reader Developed by the China Central Television (CCTV)
}

\author{
Yang Shimin ${ }^{1,2 *}$ Wu Qiong ${ }^{2}$ \\ 1Jiangxi Normal University, Nanchang, Jiangxi Province, China, 330027; \\ 2Jiangxi Normal University Science and Technology College, Gongqing, Jiangxi Province, \\ China, 332020. \\ ${ }^{*}$ Corresponding author
}

Key Words: The Reader, Culture and Sentiment, TV Program.

\begin{abstract}
In the contemporary age of cross-media integration, programs categorized as variety shows and cultural programs are generally plagued by the tendency of pan-entertainment. Against such a backdrop, TV Broadcasters are required to tread on a path that strikes a balance between viewership rating and quality, and between revenue and sentiment. The Reader developed by the China Central Television (CCTV) is indeed an attempt of exploring such a path that features innovation, integration and lead. The Reader is a large-scale show categorized as a cultural and sentimental program that features "People, Telling, Feeling, Performance and Delivery". Through the method of presentation that features a person, a feeling, an article and a pavilion, the program aims to encourage readers to present the value behind the words with the most ordinary voice. In the online and offline reading pavilions, everyone could feel the power brought along by the words. Such a "diverse" combination embodies the production team's attempt of exploring and innovating the form of the program.
\end{abstract}

\section{Introduction}

As the multi-screen era comes against the backdrop of the cross-media integration, people nowadays are inclined to acquire information and watch videos in a fragmented way. Programs that cater to such a viewing habit have taken the place of the conventional media forms and mainstream show patterns developed by the traditional media. Specifically, shows that are "self-made by networks" (i.e., produced by the video networks themselves) are constantly emerging in large quantity. As such shows feature an "brand-new, star-studded, rustically tempting and sex-related" style that exceeds the limit and caters to the entertainment, they have attracted a lot of "fans". Against such a backdrop, it has now become an imminent issue for the traditional media to leverage their strengths to rejuvenate the mainstream consciousness and culture. They are required to identify the ways of forging a batch of TV programs that ensure both the viewership rating and the quality and strike a balance between the revenue and the sentiment.

\section{Innovating the Forms of the Cultural and Sentimental Programs}

The Reader is the first TV program in which Dong Qing acts as a producer. The program is jointly developed by CCTV Innovation Media Co., Ltd and TVZONE Media and Communication Co., Ltd. As the representative production team of the CCTV group and the Hunan provincial broadcasters, both of the media companies have rich experience in producing TV programs. They have produced a large amount of programs that win universal praise, such as Defying the Impossible, Joy Street, Great Love of the East, Soldiers Sortie and Speak up for My World. Such a rich production experience has ensured the quality of the program of The Reader, and in the meantime, both of the powerful TV program producers have injected the uniqueness into the show through the exchange 
of views. Together, they have presented their thinking on the technical expression and the cultural heritage as players of the media industry, and innovated the forms of the program in various aspects.

\subsection{Innovating the Show Patterns}

In recent years, traditional media groups have successively developed a series of cultural TV programs such as The Chinese Idiom Conference, The Chinese Poetry Conference and Letters Live. Among them, the former two shows are categorized as cultural and intellectual programs of a competitive nature, whereas the latter one is a reading promotion program that features the form of stars reading the letters. The inner links of these programs are professional to a certain extent, and are rather demanding for the depth and breadth of the general audience's cognitive capacity. On the contrary, the program of The Reader attempts to "identify the values behind the words with the simplest and the most unadorned emotion" [1], and focuses on the core links between the "reading" and the "reader".

There are twelve episodes of every season of The Reader with each episode featuring a key word and five or six pairs of guests. Based on the reading of literary works, the program taps into the feelings of the "reader" through interview. Guests are invited to tell a story, an experience or some of his feelings during their life so that they could be connected emotionally with the literary works ready to be read aloud. Through the form of reading, they reveal their innermost feelings to the audience so that they could "present the sentiment of a real figure in the flesh and soul" [2]. The program is both an innovative attempt to renew the forms of cultural TV programs and an awakening call of the deficient interpersonal sentiments in current days.

\subsection{Innovating the Stage Art of the Studio}

As The Reader is a large-scale cultural and sentimental program recorded in the studio, the stage art and set design holds key to the display of the word "culture". Jorge-Luis Borges, the renowned blind writer, once described the paradise in his writing. He wrote: "The heaven has granted me a vast pile of books and a pair of eyes that could not see. Despite that, I have still been imagining secretly that the heaven should be like a library." For the readers, the library shall also be like the paradise. Designers of the studio of The Reader have apparently been inspired such a concept. The stage is set against the real exteriors of the bookshelves and racks of the library, whereas the auditorium is designed to accommodate double-layer seats like a grand theatre. The stage is connected to the auditorium seamlessly with beams to fill the building with aesthetic beauty. As for the interview zone that rather requires privacy, it is connected with the stage through the "door" located in the middle of the bookshelves. The interview zone seems to convey a sense of exploring the secrets hidden in the books. The guests are interviewed by the hosts there so as to fulfill the rendering of feelings in an explicit way that matches the key word of the specific episode. All these detailed features of design and display have added to the cultural content of the program.

\subsection{Innovating the Composition of the Guests Invited to the Program}

In terms of the composition of the guests, The Reader adopts the method of including "ordinary people, celebrities and stars". The invited guests include the newly selected ordinary people, the celebrities from all walks of life and the stars commonly seen in TV shows.

In the era of pan-entertainment, it has become un undisputable fact that programs rely on the celebrity effect to win sponsorship and the market's attention prior to the broadcast. As stars have received professional training, they outperform ordinary people in terms of both the stage performance and the mastery of reading techniques. Including stars into the program may add to the market's attention while meeting the needs of the audience for experiencing the aesthetic concept of "reading".

As a cultural and sentimental program, The Reader has a unique position. The producer Dong Qing once said that: "We have forsaken the invitation of an all-star list of guests and decided to 
include ordinary people to the program, who can always bring us surprise and causes for being moved. The readers are required to have rich experience, touching stories and real sentiments, or so to speak, a special quality that is worth promotion" [3]. Finally, "celebrities" from all walks of life. They have the double identity of being both the ordinary people and the celebrities of the industry. Therefore, they could read on behalf of themselves while at the same time, they could represent the community of an entire industry. Thus they are more likely to trigger resonance from a larger audience. However, their delivery proves to be sufficient for the audience to forget about the patterns and set aside the linguistic expression, while being deeply immersed in the sentimental stories behind the words.

\section{Media Convergence against the Backdrop of Cross-media Integration}

Subsequent to the broadcast of The Reader, a heated debate has quickly taken place among the audience. Besides the quality production of the program itself, the media convergence of the program is also a topic worth mentioning.

\subsection{Interactive Media Convergence Both Online and Offline}

The "reading pavilions" established both online and offline are undoubtedly the best extension for the program to interact with the audience. The production team of the program has set up the online "reading pavilions" through the channel of the WeChat Public Account to make it easier for the pubic to share their feelings and reading. In this way, the audience has gained a brand-new experience of interaction. The offline "reading pavilions" are spread across the campus, communities and downtown areas so that people may express their feelings through reading freely. Such pavilions are equipped with simple recording equipment, and in the meantime, readers will find it easier to express the sentiments behind the words in a completely independent space, thus experiencing the unique power brought along by reading. Before the hosts come to the stage in each episode, videos will be selected among those in the recording library of the reading pavilions to be broadcasted. Such a method not only ensures the interactive communication both online and offline, but also inspires the audience's passion for reading and enhances the loyalty of the readership.

\subsection{Multi-screen Fusion and Mutually Assisted Communication}

To meet the diversified needs of the audience for the program, The Reader adopts the method of multi-screen fusion and mutually assisted communication through "Twin accounts of Weibo and WeChat, Twin Satellite Channels and Triple Video Websites". Namely, the program reaches its audience through the official accounts of Weibo and WeChat, the Channels of CCTV1 and CCTV3 along with the video websites of CCTV, Tencent and iQiyi.

As of May 22nd, 2017, The Reader had published a total of 1486 posts through its official Weibo Account and 312 articles with over 100,000 page views through its WeChat Public Account[4]. The content of the program was introduced through various forms of communication such as texts, pictures and small videos. The audience may also accurately locate and watch the video clips of the program in which they are interested based on their preferences. The model of the multi-screen fusion and mutually assisted communication has met the diversified needs of the audience. In the meantime, such a model is conducive to disseminating the fragmented information in current days and promoting the program.

\section{Conclusion}

The Reader is an original cultural program broadcasted among the first batch of shows launched in CCTV's channels in 2017. The program features precise positioning, a powerful list of guests, moving stories and superb production quality. Hence, it has been widely recognized by the majority of the audience and become a "phenomenal" cultural program. The program has won both good 
reputation and high viewership. However, although the Chinese culture is profound and immense, it is not easy for a cultural program to succeed. The broadcasters are not only required to conduct an in-depth study of the cultural connotations, the audience expectations and the rules of communication for the program, but also to undertake the media responsibilities for spreading mainstream culture and rejuvenating the lost classics. In a nutshell, The Reader developed by CCTV has showcased the renewed reflections on the original cultural programs by traditional media people. In addition, the program has offered a new direction for related parties to transform and upgrade the TV programs and even the online video programs.

\section{Acknowledgement}

Funding: Study on the Self-made Programs of Video Websites, a research project on the Humanities and Social Sciences at the university level of Jiangxi Province (YS162044); one of the phased outcomes of the Research on the Status-quo and Prospects of the Programs "Self-made by Networks" against the Backdrop of Cross-media Integration (YG2016211), a project under the Cultural and Artistic Sciences Program of Jiangxi Province.

\section{References}

[1][2] The introduction of the program of The Reader, http://tv.cctv.com/lm/ldz/.2017.2.18.

[3] Dong Qing: Stars have been over-consumed, and ordinary people will bring us more surprises if invited to the program. http://www.hn.xinhuanet.com/2017-02/22/c_1120508293.htm.
[4]The
official
Weibo
Account
of
The
Reader,
http://photo.weibo.com/6000208460/wbphotos/large/mid/4111021104880321/pid/006y4gjOly1ff wpc2vtf3j30zk0jyn0c. 